\title{
Dissociative production of vector mesons as a new tool to study gluon saturation at electron-ion colliders
}

\section{Bendova*, J. Cepila, and J. G. Contreras}

Faculty of Nuclear Sciences and Physical Engineering, Czech Technical University in Prague, Prague 11519, Czech Republic

E-mail: dagmar.bendovalfjfi.cvut.cz, jan.cepila@fjfi.cvut.cz, jgcn@mail.cern.ch

\begin{abstract}
We present predictions for the dissociative photo- and electroproduction of light and heavy vector mesons off protons. The computation is based on the energy-dependent hot-spot model which incorporates geometric fluctuations of the target-proton partonic structure in the impact-parameter plane. We observe a striking feature of the dissociative cross section as a function of the center-ofmass energy of the photon-proton system. The cross section presents a maximum, whose position depends on the mass of the vector meson and the virtuality of the exchanged photon. By using the positions of these maxima, we define a geometric saturation scale. We find that this scale grows linearly with energy as a function of the scale of the process and we discuss the possibility to map this scale experimentally with respect to the kinematical reach of the proposed electron-ion colliders, JLEIC, eRHIC, and LHeC. This contribution presents results published in Phys. Rev. D 99, 034025 (2019).
\end{abstract}

Light Cone 2019 - QCD on the light cone: from hadrons to heavy ions - LC2019 16-20 September 2019

Ecole Polytechnique, Palaiseau, France

\footnotetext{
${ }^{*}$ Speaker.
} 


\section{Introduction}

Quantum chromodynamics (QCD) predicts a linear evolution of the partonic structure of hadrons with energy. However at some point, this behavior is predicted to change with the onset of nonlinear effects and the hadron subsequently enters a so-called saturated regime. The production of vector mesons has been advocated as a good probe of saturation effects at the LHC [1] and at planned EIC facilities [2,3]. In the dissociative process, the incoming electron emits a photon. This photon, which can be quasi-real or it can have a large virtuality $Q^{2}$, interacts with the target proton. Subsequently, a vector meson is produced and the proton is broken into a hadronic system. This process can be related to the fluctuations of the partonic structure of the proton within the Good-Walker approach $[4,5]$. This approach was followed in [1] and using a so called energydependent hot-spot model, the authors shown that the total dissociative $\mathrm{J} / \psi$ photoproduction cross section presents a maximum as a function of the center-of-mass energy $W_{\gamma p}$ of the photon-proton system. The scale dependence of the photo- and electroproduction for various vector mesons has been studied in [6]. This contribution reviews the predictions of [6] for the geometrical saturation scale defined by the maxima of dissociative vector meson cross section. The text is organized as follows. In Sec. 2 we summarize the formalism of the dissociative vector meson production within the energy-dependent hot-spot model, in Sec. 3, we present some selected predictions of our model for dissociative vector meson cross sections and compare them with the available experimental data. In this section, we also discuss the predicted geometrical saturation scale, and in Sec. 4, we give a brief conclusion.

\section{Dissociative production of vector mesons in an energy-dependent hot-spot model}

The dissociative cross section to produce a vector meson $\mathrm{V}$ is given as a variance over different target configurations (for details and a full list of references see [6])

$$
\left.\frac{\mathrm{d} \sigma^{\gamma^{*} p \rightarrow \mathrm{VY}}}{\mathrm{d}|t|}\right|_{T, L}=\frac{\left(R_{g}^{T, L}\right)^{2}}{16 \pi}\left(\left\langle\left|\mathscr{A}_{T, L}\right|^{2}\right\rangle-\left|\left\langle\mathscr{A}_{T, L}\right\rangle\right|^{2}\right)
$$

where $\mathscr{A}_{T, L}$ is the photon-proton scattering amplitude which can be expressed as

$$
\mathscr{A}_{T, L}\left(x, Q^{2}, \vec{\Delta}\right)=i \int \mathrm{d} \vec{r} \int_{0}^{1} \frac{\mathrm{d} z}{4 \pi} \int \mathrm{d} \vec{b}\left|\Psi_{\mathrm{V}}^{*} \Psi_{\gamma^{*}}\right|_{T, L} \exp [-i(\vec{b}-(1-z) \vec{r}) \vec{\Delta}] \frac{\mathrm{d} \sigma_{q \bar{q}}}{\mathrm{~d} \vec{b}},
$$

where $x$ is the momentum fraction of the exchanged pomeron, $\vec{r}$ is the transverse size of the color dipole, and $\vec{b}$ is the impact parameter. In the impact-parameter factorized form, the dipole-proton cross section is $\frac{\mathrm{d} \sigma_{q \bar{q}}}{\mathrm{~d} \vec{b}}=\sigma_{0} N(x, r) T_{p}(\vec{b})$, where $T_{p}(\vec{b})$ is the function which describes the proton profile in the transverse plane, $\sigma_{0}$ is a normalization parameter, and $N(x, \vec{r})$ is the dipole-proton scattering amplitude with the prescription given by the Golec-Biernat and Wusthoff model [7, 8].

In the energy-dependent hot-spot model [1], the proton is seen as a set of hot spots (regions with high gluon density). The proton profile function describes the positions of the hot spots in the impact-parameter plane as

$$
T_{p}(\vec{b})=\frac{1}{N_{h s}} \sum_{i=1}^{N_{h s}} T_{h s}\left(\vec{b}-\vec{b}_{i}\right) ; \quad T_{h s}\left(\vec{b}-\vec{b}_{i}\right)=\frac{1}{2 \pi B_{h s}} e^{-\frac{\left(\vec{b}-\vec{b}_{i}\right)^{2}}{2 B_{h s}}},
$$


where $B_{p}$ and $B_{h s}$ are related to the mean square radius of the proton and hot spot, respectively. The energy-evolution of the proton structure is ensured by the number of hot spots $N_{h s}$ increasing with decreasing $x$. The positions in $b$ and number of hot spots fluctuate event-by-event. For the detailed description of the model and it's parameters, see [1, 6, 9].

\section{Predictions of the model}

\subsection{Dissociative production of vector mesons compared with data}

We predict an energy $W_{\gamma p}$ dependence of the dissociative photo- and electroproduction of vector mesons off the proton target using the above briefly introduced model. In this contribution, we show the predictions for $\rho$ and $\mathrm{J} / \psi$ mesons and compare them with the available experimental data. The predictions for other vector mesons as well as for the exclusive process are listed in [6].

Figure 1 shows the predictions of the model for the energy $W_{\gamma_{p}}$ dependence of the dissociative cross section for $\rho$ (left) and $\mathrm{J} / \psi$ (right) vector meson production. Predictions at different virtualities are compared with HERA data $[10,11,12]$ showing a good agreement in all cases.

\subsection{Geometrical saturation scale}

As it has been observed in [1], the total dissociative cross section at first grows with increasing energy $W_{\gamma_{\mathrm{p}}}$. However, this growth stops at some point and when going to even higher energies, the cross section decreases as a function of $W_{\gamma \mathrm{p}}$. This phenomenon is clearly present for various vector mesons (for full list see [6]). We have also observed that the position of this maximum as a function of $W_{\gamma_{\mathrm{p}}}$ is shifted towards higher energies as the mass of the vector meson and $Q^{2}$ of the exchanged photon increases.

This behavior is caused by the form of the cross section in Eq. 2.1. The dissociative process is sensitive to the variance over different configurations of the fluctuating proton structure. As the number of hot spots, which have the same size in the transverse plane in our model, grows with
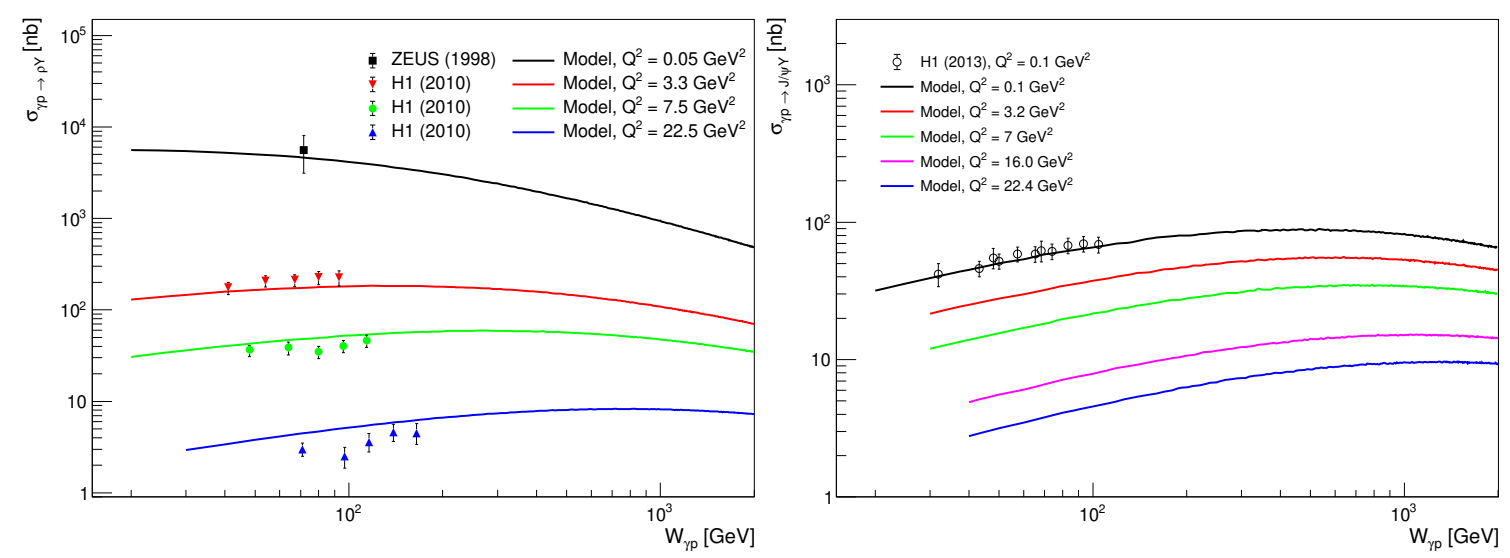

Figure 1: Comparison of the model predictions (solid lines) with the experimental data for the $W_{\gamma p}$ dependence of the dissociative photo- and electroproduction cross section of vector mesons. Left: Predictions for a $\rho$ meson compared with HERA data [10, 11]. Right: Predictions for a $J / \psi$ meson compared with H1 [12] data. Figures taken from [6]. 
increasing $W_{\gamma \mathrm{p}}$, the proton area is being filled. At some point, the available area of the proton is filled with overlapping hot spots and all the possible configurations start to look alike which causes the variance to start decreasing. The maxima of the dissociative cross section are located at a well defined point in $W_{\gamma_{\mathrm{p}}}$ at a given scale of the process - these points form a scale which we call the geometric saturation scale. In general, this scale can have different behavior than a saturation scale $Q_{s}(x)[7,8]$ and in the following, we discuss its origin and some of its properties studied in [6].

We show this scale in Fig. 2. The points represent the energy $W_{G S S}$ (left plot) and related $x_{G S S}$ (right plot) at which the maxima are located for each vector meson, defined by its mass $M^{2}$, and for each virtuality $Q^{2}$ of the exchanged photon. The value at the maximum can fluctuate because the predictions are based on a random process. We take into account these fluctuations by taking a region containing $1 \%$ of the largest cross section values to determine the location of the maximum and to estimate the associated uncertainty.
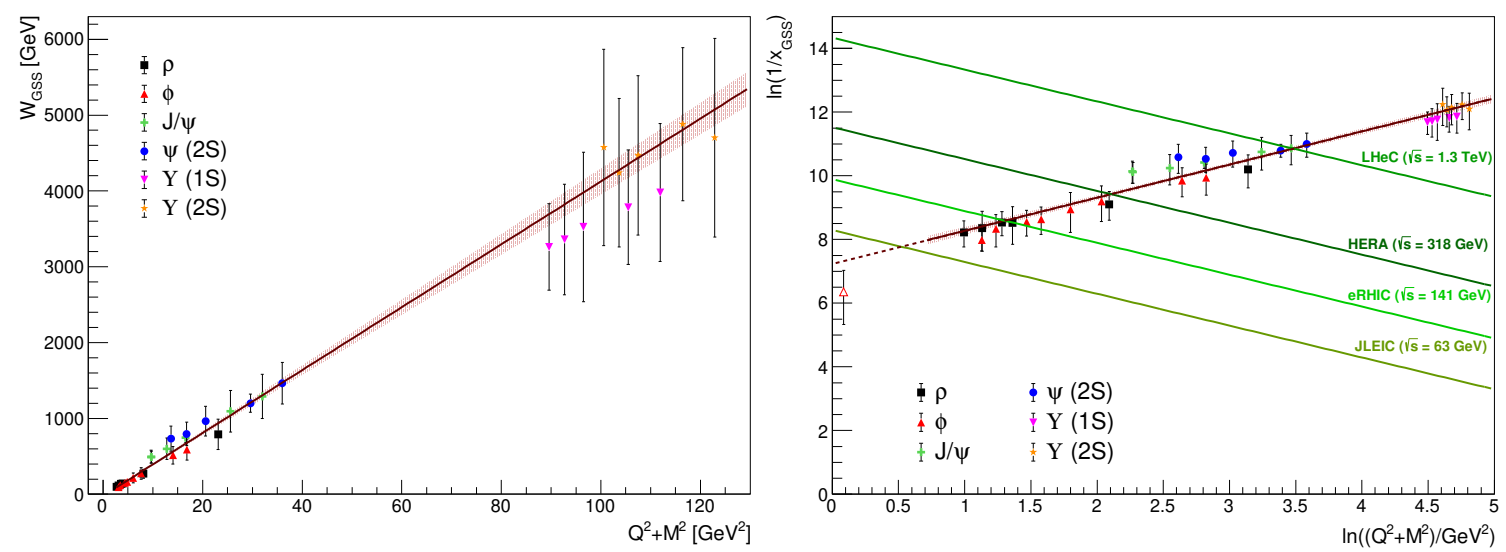

Figure 2: Left: Position of the maxima of the dissociative cross sections (markers) and an estimation of the related uncertainty (bars) as a function of $Q^{2}+M^{2}$. Right: the same data as in the left panel, but translating $W_{\mathrm{GSS}}$ into $x_{\mathrm{GSS}}$. The green lines represent the kinematic reach of some of the proposed future electron-ion colliders. The red lines in both plots are fits to the extracted maximum position, for details see [6].

We find that the dependence of $W_{G S S}$ on $Q^{2}+M^{2}$, which represents the measure of the scale of the process, presents a linear behavior. This linear behavior is also present in logarithmic $1 / x_{G S S}$ vs $Q^{2}+M^{2}$ plot. In the same figure, we show the kinematic reach of some of the proposed future electron-ion facilities. It is very interesting to see that this phenomenon could be measured at all the proposed facilities for various vector mesons. The envisaged capabilities of the proposed JLEIC and eRHIC detectors could be able to investigate the positions of the maxima for light vector mesons $\rho$ and $\phi$ at relatively low virtualities. To measure this phenomenon for photoand electroproduction of $\mathrm{J} / \psi$, one needs the capabilities of $\mathrm{LHC}$ and $\mathrm{LHeC}$, while heavier vector mesons like $\Upsilon(1 S)$ or $\Upsilon(2 S)$ are beyond the reach of the proposed electron-ion colliders.

\section{Conclusions}

In this contribution, we have presented the predictions for the total dissociative photo- and electroproduction of vector mesons $\rho$ and $\mathrm{J} / \psi$ off protons using the energy-dependent hot-spot model. We observe a striking behavior of the dissociative cross section as a function of energy 
$W_{\gamma_{\mathrm{p}}}$ - the cross section rises at low energies and at some point, it reaches a maximum and decreases afterwards. This phenomenon can be tracked down to the variance over different proton configurations in the impact parameter plane - when going to low $x$, the hot spots start to overlap which causes the possible configurations to look alike and therefore, the variance decreases. We use these maxima to define the geometric saturation scale which we find to follow a linear behavior in $\ln \left(\frac{1}{x_{G S S}}\right)$ with respect to the logarithmic scale of the process. This phenomenon is caused by the geometric fluctuations of the proton in the transverse plane and the arising geometric saturation scale could be mapped at future EIC facilities.

\section{Acknowledgements}

This work has been supported by the COST Action CA15213 THOR, by the grant LTC17038 of the INTER-EXCELLENCE program at the Ministry of Education, Youth and Sports of the Czech Republic, and Grant No. 17-04505S of the Czech Science Foundation (GACR).

\section{References}

[1] J. Cepila, J. G. Contreras and J. D. Tapia Takaki, Energy dependence of dissociative J/ $\psi$ photoproduction as a signature of gluon saturation at the LHC, Phys. Lett. B766 (2017) 186 [1608.07559].

[2] A. Accardi et al., Electron Ion Collider: The Next QCD Frontier, Eur. Phys. J. A52 (2016) 268 [1212.1701].

[3] LHeC Study GRoup collaboration, A Large Hadron Electron Collider at CERN: Report on the Physics and Design Concepts for Machine and Detector, J. Phys. G39 (2012) 075001 [1206. 2913].

[4] M. L. Good and W. D. Walker, Diffraction disssociation of beam particles, Phys. Rev. 120 (1960) 1857.

[5] H. I. Miettinen and J. Pumplin, Diffraction Scattering and the Parton Structure of Hadrons, Phys. Rev. D18 (1978) 1696.

[6] D. Bendova, J. Cepila and J. G. Contreras, Dissociative production of vector mesons at electron-ion colliders, Phys. Rev. D99 (2019) 034025 [1811.06479].

[7] K. J. Golec-Biernat and M. Wusthoff, Saturation effects in deep inelastic scattering at low $Q^{* * 2}$ and its implications on diffraction, Phys. Rev. D59 (1998) 014017 [hep-ph/ 9807513 ].

[8] K. J. Golec-Biernat and M. Wusthoff, Saturation in diffractive deep inelastic scattering, Phys. Rev. D60 (1999) 114023 [hep-ph/9903358].

[9] J. Cepila, J. G. Contreras, M. Krelina and J. D. Tapia Takaki, Mass dependence of vector meson photoproduction off protons and nuclei within the energy-dependent hot-spot model, Nucl. Phys. B934 (2018) 330 [1804.05508].

[10] ZEUS collaboration, Elastic and proton dissociative $\rho^{0}$ photoproduction at HERA, Eur. Phys. J. C2 (1998) 247 [hep-ex/9712020].

[11] H1 collaboration, Diffractive Electroproduction of rho and phi Mesons at HERA, JHEP 05 (2010) 032 [0910.5831].

[12] H1 collaboration, Elastic and Proton-Dissociative Photoproduction of J/psi Mesons at HERA, Eur. Phys. J. C73 (2013) 2466 [1304.5162]. 